\title{
Development of immunotherapy for brain metastasis (Review)
}

\author{
RUILIN DING ${ }^{1 *}$, LONGXIA CHEN $^{1 *}$, ZHOU SU $^{2}$, TENGQIONG XIONG ${ }^{1}$, \\ QINGLIAN WEN ${ }^{3}$, QING PENG ${ }^{4}$ and FENG JIANG ${ }^{4}$ \\ ${ }^{1}$ Institute of Drug Clinical Trial/GCP Center, Affiliated Hospital of Southwest Medical University, Luzhou, Sichuan 646000; \\ ${ }^{2}$ Department of Oncology, Sichuan Mianyang 404 Hospital, Mianyang, Sichuan 621000; ${ }^{3}$ Department of Oncology, \\ Affiliated Hospital of Southwest Medical University, Luzhou, Sichuan 646000; ${ }^{4}$ Department of Cardiology, \\ Affiliated Hospital of Southwest Medical University, Luzhou, Sichuan 646000, P.R. China
}

Received January 30, 2020; Accepted June 22, 2020

DOI: 10.3892/ijo.2020.5091

\begin{abstract}
Brain metastasis (BM) is associated with a poor prognosis, with the typical overall survival rate ranging from weeks to months in the absence of treatment. Although the concept of immune privilege in the central nervous system has eroded over time, the advent of immunotherapy has opened a new set of potential therapeutic options for patients with BM. Recently, immunotherapy has been demonstrated to confer survival advantages to patients with multiple malignancies commonly associated with BMs. Data from a number of clinical trials have demonstrated that immune checkpoint inhibitors are effective for patients with BM. In addition, cellular therapies, including the application of chimeric antigen receptors T-cell therapy and dendritic cell vaccine, have also been utilized in the treatment of BM. In the present review, preclinical and clinical evidence supporting the applicability of immunotherapy for the treatment of BMs from melanoma, non-small cell lung cancer (NSCLC) and renal cell carcinoma (RCC) were examined, where the challenges and safety of this treatment modality were also discussed.
\end{abstract}

\section{Contents}

1. Introduction

2. Biology of brain metastases

3. Evidence of treatment with ICIs for patients with BMs

4. Cellular therapy for patients with BMs

5. Conclusions

Correspondence to: Dr Ruilin Ding, Institute of Drug Clinical Trial/GCP Center, Affiliated Hospital of Southwest Medical University, 25 Taiping Street, Luzhou, Sichuan 646000, P.R. China

E-mail: rainingdean@163.com

*Contributed equally

Key words: immunotherapy, brain metastasis, immune checkpoint inhibitors, cellular therapy, review

\section{Introduction}

Brain metastases (BMs) occur in $\leq 20 \%$ adults with systemic malignancies, which represent a significant clinical challenge $(1,2)$. Patients who develop BMs have a poor prognosis, with an average survival rate of $<6$ months (3). Although surgery, chemotherapy and radiotherapy (RT) represent the mainstay of treatment options for patients with BMs (4), such treatments are rarely curative and result in substantial toxicity owing to the sensitivity of the brain (5).

T-cell immunotherapy has demonstrated promising early results in the treatment of patients with BM, which has challenged the traditional paradigm that the brain is an organ with immune privilege (2). A number of approaches have been applied to stimulate or enhance endogenous T-cell immune responses for brain tumors or BMs, including tumor neoantigen vaccines (6), chimeric antigen receptor (CAR) T-cells (7) and immune checkpoint inhibitors (ICIs) $(8,9)$. Among these, ICIs have become the protagonist in anticancer immunotherapy. The most clinically relevant ICIs are those targeting the programmed cell death protein 1 (PD-1) and its ligand programmed cell death ligand (PD-L)1, in addition to cytotoxic T-lymphocyte-associated protein 4 (CTLA-4). CTLA-4 and PD-1 are both the members of the B7/CD28 family; however, their inhibitory effects are mediated via different pathways. The blockade of CTLA-4 prevents the reception of inhibitory signals from B7 during the priming phase of $\mathrm{T}$ cell activation. By contrast, anti-PD-1 therapies block the negative regulation of cytotoxic $\mathrm{T}$ lymphocytes during the effector phase by preventing their interaction with PD-L1 and PD-L2 (10-12). Clinical studies have previously suggested that ICIs are effective in improving the overall survival (OS) in patients with BMs $(8,9)$, though these observations were never confirmed by large-scale prospective phase III clinical studies. Additionally, the engineering of new T-cell immunity using CARs is a cancer treatment strategy that is rapidly advancing in this field $(13,14)$. For patients with brain tumors or BMs, preliminary results with CAR T-cell therapy have also shown promise.

Therefore, in the present review, existing preclinical and clinical evidence that supports the applicability of immunotherapy for the treatment of BMs, including that the use of ICIs and cell immunotherapy, were enumerated. Additionally, 
the challenges and safety of this treatment modality were discussed.

\section{Biology of brain metastases}

The brain was previously considered to be an organ with immune privilege that is excluded from systemic immune surveillance (10). The presence of the blood-brain-barrier (BBB) and the absence of lymphatic vessels, which serve as a highway for antigen presenting cells (APC), may have contributed to this concept (4).

In the healthy brain, specialized tight junctions between endothelial cells in the BBB impede communication with the circulatory system (15), restricting the entry of immune cells from the peripheral circulation. However, it has recently been revealed that resting $\mathrm{T}$-cells can migrate from the meningeal blood vessels into the cerebrospinal fluid (CSF) (16). In addition, under various pathophysiological conditions, the integrity of the BBB becomes compromised. The increased permeability of the $\mathrm{BBB}$ in association with pathologically impaired microvessels has been previously observed (17). Activated circulating $\mathrm{CD}^{+} \mathrm{T}$-cells have been demonstrated to cross the BBB and induce local $\mathrm{T}$ cell activation (18). Previously considered to be devoid of lymphatic vessels, a lymphatic system within the central nervous system (CNS) was discovered in 2015 (19). These findings demonstrate that T-cells can in principle cross the BBB where they can serve a role in $\mathrm{BMs}$, which have been reported by previous preclinical studies $(20,21)$.

To date, several studies have demonstrated that large quantities of tumor infiltrating lymphocytes (TILs) can be found in BMs of different primary malignancies. TIL subsets of $116 \mathrm{BM}$ specimens were investigated in a study previously conducted by Berghoff et al (22) using immunohistochemistry, which revealed that $\mathrm{CD}^{+}$and $\mathrm{CD}^{+}$TILs were present in $115 / 116(99.1 \%)$ and $112 / 116(96.6 \%)$ of the tumor specimens, respectively (22). Furthermore, 19/67 (28.4\%) of the specimens evaluated for PD-L1 expression had shown $>5 \%$ membranous expression (22). In another study, Kluger et al previously demonstrated that greater $\mathrm{CD}^{+} \mathrm{T}$ cell infiltration was associated with the delayed onset of melanoma brain metastasis (MBM) and an improved survival (23). Therefore, the observed concordance of a high TIL density with an improved OS supports the application of immunotherapy in treating patients with BMs (24).

Collectively, these aforementioned studies suggest that T-cells can move from the meningeal blood vessels into the CSF and cross the BBB in the presence of BMs. Significant TILs have been observed in BMs of different primary cancers, where high TIL density modulates the mode of immune response elicited by metastatic brain tumor cells in conjunction with other resident cell types in the brain.

\section{Evidence of treatment with ICIs for patients with BMs}

Immune checkpoint proteins, such as PD-1 and CTLA-4, are co-receptors that promote tumor cell survival through immune evasion. PD-1 is a receptor that is expressed on the surfaces of T-cells that binds to PD-L1 and PD-L2 expressed on (APCs) or tumor cells, in turn reducing T-cell activity and subsequently limiting cancer cell elimination (25). CTLA-4 is an inhibitory receptor that interacts with human leukocyte antigen (HLA)-B7-1 and HLA-B7-2 on T-cells to inhibit the initial stages of T-cell activation (2). ICIs were designed to interrupt these immunosuppressive processes and reinvigorate antitumor immune responses (26). Previous clinical trials performed, including that of EORTC 18071 (27), KEYNOTE-001 (28) and CheckMate 057 (29), revealed that using CTLA-4-based or PD-1-based ICIs can improve the survival of patients with various types of cancer, including melanoma, non-small cell lung cancer (NSCLC) and Hodgkin's lymphoma (30-32). Notably, a number of clinical studies have also been conducted on patients with BMs, which have demonstrated promising results.

Clinical data on ICIs for the treatment of BMs. There are numerous studies available that have retrospectively investigated patients with BMs who have been treated with ICIs. Among these, the largest study was conducted by Iorgulescu et al (8), which used the National Cancer Database to analyze data from 220,439 patients diagnosed with melanoma from 2010 to 2015. They found that ICIs could improve OS to 12.4 months, compared with 5.2 months for patients who did not receive ICIs. In addition, single-institution studies have also investigated the efficacy of ICIs for patients with BMs. In one such study, 128 patients with BMs secondary to NSCLC, renal cell carcinoma (RCC) and melanoma were included, where they were treated with ICIs. The 1-year survival rates for patients with NSCLC, melanoma and RCC were found to be $48.3,54.5$ and $55.4 \%$, respectively (2). Lanier et al performed a retrospective study consisting of 271 patients with BMs who were treated with stereotactic radiosurgery (SRS) (33). Of these patients, the median OS of the 101 (37\%) who received immunotherapy was 15.9 months, whilst that of the $170(63 \%)$ who did not receive immunotherapy was 6.1 months (33).

By contrast, the number of prospective clinical trials in this field remains limited. Patients with untreated or active BMs have always been excluded from clinical trials, as in the majority of cases, the management of BM requires supportive approaches, such as corticosteroids to relieve intracranial pressure (34). Table I outlines a subset of prospective trials that investigated the use of ICIs for the treatment of BMs.

The first prospective clinical trial that specifically investigated the use of ICIs in BMs was a phase II trial (trial no. NCT00623766) conducted by Margolin et al (35). The study enrolled 72 patients with both symptomatic and asymptomatic MBMs, where all patients received 4 doses of ipilimumab, an anti-CTLA4 monoclonal antibody. After 12 weeks, 9/51 (18\%) of the patients in the asymptomatic group exhibited disease control, compared with $1 / 21$ patients $(5 \%)$ in the symptomatic group (35). Ipilimumab appears to be more effective for patients with asymptomatic BMs. Another previous phase II study (NIBIT-M1) included 20 patients with asymptomatic BMs for investigating the efficacy of ipilimumab combined with fotemustine, which demonstrated that two patients achieved intracranial complete responses (CR) and 10/20 patients achieved disease control (36). Additionally, the median OS was 12.7 months, demonstrating the long-term efficacy of this combination therapy in patients with BMs (9). An expanded access program (EAP) in Italy analyzed 146 patients 


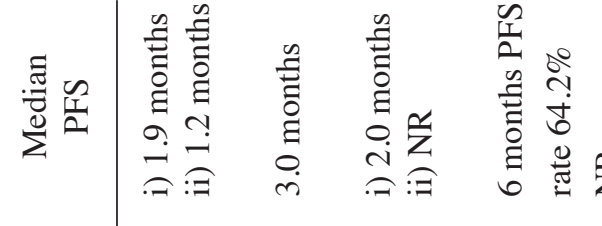

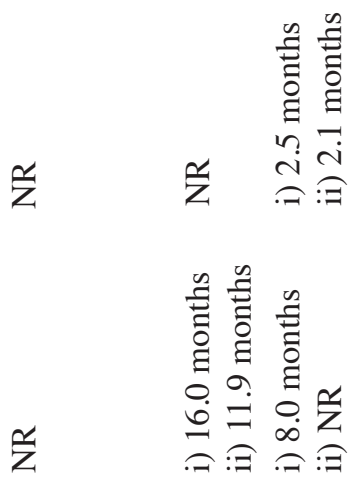

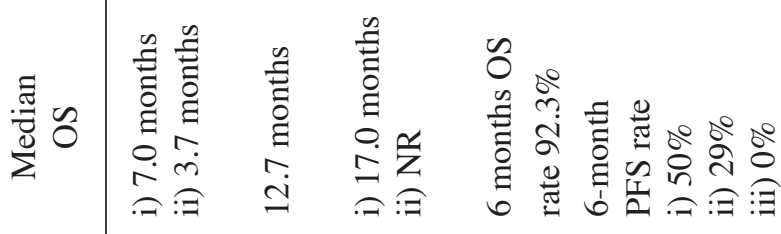

光 $\cong \cong: \cong$

.

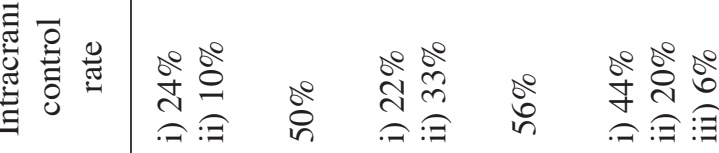

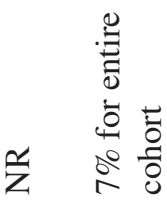

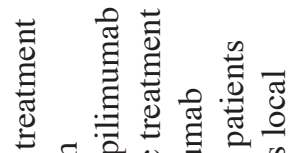

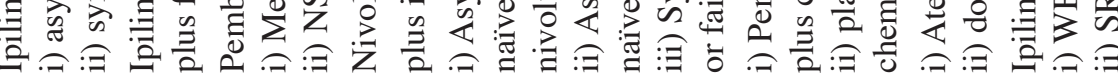

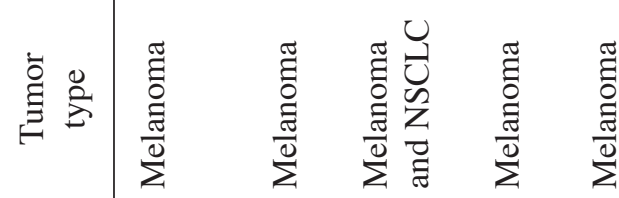

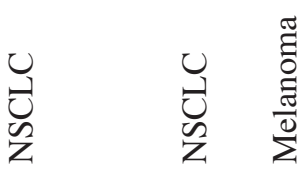

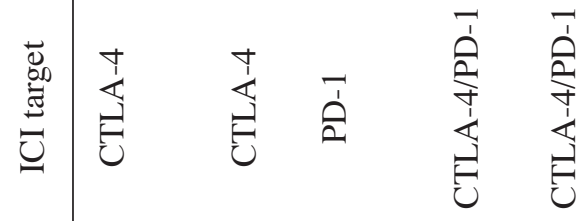

仓े

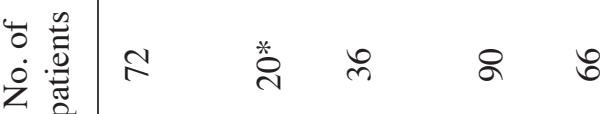

$\stackrel{*}{\infty} \stackrel{*}{\check{c}}$

家完宽

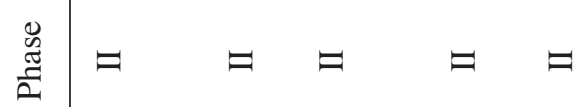

ヨ $\quad$ ヨ

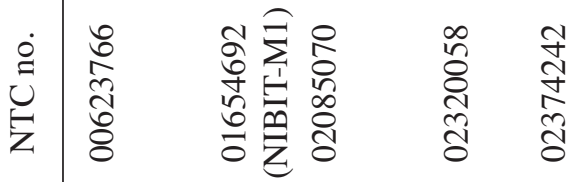

$\&$
$\&$
$\infty$
$\infty$
$i$
0

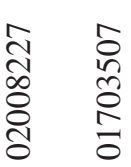


with MBMs who were treated with ipilimumab (37). That study found that 4 patients achieved CR and 13 patients had a partial response (PR) yielding a disease control rate of $27 \%$. In terms of prognosis, $\sim 20 \%$ of the patients were alive 1 year after the initiation of ipilimumab treatment, where the median progression-free survival (PFS) and OS were revealed to be 2.8 and 4.3 months, respectively (37).

The first anti-PD-1 monoclonal antibody that clearly demonstrated efficacy against untreated BMs was pembrolizumab created by Merck \& Co Inc. Patients with melanoma or NSCLC with measurable BMs $(>5-20 \mathrm{~mm})$ were enrolled into a phase II study to evaluate the efficacy of pembrolizumab in both cohorts (trial no. NCT02085070) (38). Brain metastasis responses were achieved in 4 of $18(22 \%)$ patients with melanoma and 6 of 18 patients (33\%) with NSCLC, according to the interim analysis of this study (38). The final results of the full melanoma cohort revealed that $26 \%$ patients exhibited a BM response, where the median PFS and OS were 2 and 17 months, respectively. The 2-year survival rate was similar compared with that in patients without BMs treated with the anti-PD-1 antibodies, suggesting that pembrolizumab is effective for treating MBMs with acceptable toxicity (39). The final results of the full NSCLC cohort of that study have not yet been reported.

Additional data supporting the use of pembrolizumab for untreated BMs are derived from a study funded by Merck \& Co Inc. (KEYNOTE-189). KEYNOTE-189 (trial no. NCT02578680) compared the efficacy of pembrolizumab combined with chemotherapy and chemotherapy alone in patients with metastatic NSCLC, including 109 patients with BMs (17.5\%). In the subgroup analysis of the BM patients, the 73 patients treated with combined pembrolizumab + chemotherapy exhibited markedly longer OS compared with the 35 patients treated with chemotherapy alone (40).

Similar to pembrolizumab, nivolumab has also demonstrated efficacy for untreated BMs. In a pool analysis of data from the CheckMate 017 (trial no. NCT01642004), CheckMate 057 (trial no. NCT01673867) and CheckMate 063 (trial no. NCT01721759) studies, patients with BM treated with nivolumab showed a longer median OS (8.4 months) compared with those treated with docetaxel (6.2 months) (41). In another phase II ABC study (trial no. NCT02374242) conducted previously, 27 patients with asymptomatic MBMs were treated with nivolumab alone (42). At the data cutoff, the intracranial response rate of this population was found to be $20 \%$, where the median intracranial PFS and OS were calculated to be 2.5 and 18.5 months, respectively (42). In addition, an EAP in Italy included 409 patients with asymptomatic BMs associated with NSCLC, who were treated with nivolumab (43). The disease control rate of these patients was revealed to be $39 \%$, which included 4 and 64 patients achieving CRs and PRs, respectively. The median OS was 8.6 months. The results of this Italian EAP suggested that nivolumab is effective in patients with BMs associated with NSCLC (44). These observations regarding nivolumab treatment were consistent with another Italian EAP previously conducted, which included 389 patients with RCC, 32 of whom had BMs. The intracranial response rate of the $\mathrm{BM}$ patients was $18.7 \%$ with a disease control rate of $53.1 \%$, demonstrating that patients with BM-associated RCC may also benefit from nivolumab application (44).
The efficacy of atezolizumab, an inhibitor of PD-L1, in patients with and without a history of BMs was previously evaluated in the OAK trial (trial no. NCT02008227) (45). In patients with a history of BMs, median OS was longer in patients treated with atezolizumab compared with those treated with docetaxel (16.0 vs. 11.9 months). Furthermore, patients with asymptomatic BMs treated with atezolizumab had a lower probability of developing new symptomatic brain lesions compared with those treated with docetaxel (45).

The possibility of combining the two ICIs for the treatment of BMs has also been evaluated in several phase I/II clinical trials. CheckMate-204 (NCT02320058) is a phase II study combining nivolumab and ipilimumab to treat MBMs (46). Subsequent analysis revealed a $52 \%$ intracranial response rate, including 24 (26\%) CRs. The intracranial clinical benefit rate was $57 \%$ (46), concordant with extracranial activity. A similar result was found in the phase II ABC study (trial no. NCT02374242) aforementioned (42). The intracranial response rate in patients treated with the nivolumab + ipilimumab combination was $46 \%$, which was notably higher compared with those treated with nivolumab alone $(20 \%)$. Following a median follow-up of 14 months, both median PFS and median OS were not reached in the combination group (42). These results indicate that the combination of these two kinds of ICIs may confer superior efficacy compared with the administration of either ICI alone, where the combination therapy can be considered as the first-line therapeutic option for patients with BM.

Overall, the data reviewed as aforementioned demonstrate that the use of ICIs has significant utility for the treatment of BMs. However, the majority of these trials were early clinical studies with small sample sizes. Larger studies will be required to verify the observations of these early findings. The majority of clinical trials had enrolled patients with BM who had at least one target intracranial lesion. Thus, the treatment strategies of immunotherapy for isolated and multiple BMs are the same currently. Further studies are warranted to determine the difference between isolated and multiple BMs treatment. Nevertheless, due to previous findings that treatment with ICIs combined with other therapies has demonstrated promising efficacy for solid tumors, this intervention strategy should also be considered for BMs.

Integration of radiation therapy with ICIs for the treatment of BMs. RT is currently the standard therapeutic intervention option for patients with non-resectable BMs. Whole brain radiotherapy (WBRT) and SRS are two of the most important forms of RT. Conventionally, WBRT is prescribed for patients with multiple BMs, poor prognosis and/or poor performance status (PS), whilst SRS would be considered for patients with fewer numbers of BMs and those with good PS (47). Since SRS can achieve high local tumor control without affecting healthy brain tissues, it is associated with a reduced risk of severe side-effects compared with WBRT, including neurocognitive damage and hair loss (48).

The integration of RT with the ICIs has been investigated in patients with BMs in previous studies. The rationale for combining ICIs with RT may be derived from the previously observed effects of RT on the immune system. RT has been demonstrated to induce tumor cell death (49), resulting in the 
release of tumor-associated antigens (50), which activate APCs to then prime cytotoxic T-cells to kill tumor cells at distant locations $(51,52)$. RT has also been shown to upregulate major histocompatibility complex (MHC) I expression on tumor cells (53). Previous studies have reported that RT can alter the tumor environment, induce the secretion of proinflammatory chemotactic factors by cancer cells that mediate strong immunostimulatory effects $(54,55)$ and even upregulate PD-L1 expression in tumor cells (56), which could in theory enhance the antitumor effect of anti-PD-L1 antibodies (57).

Numerous previous studies have investigated the efficacy and safety of combining ICIs and RT for patients with BM. In particular, two similar studies retrospectively evaluated the outcomes of 46 patients with MBMs, who were treated with ipilimumab and SRS, where both revealed improved responses when SRS was administered concurrently with ipilimumab $(58,59)$. Diao et al $(60)$ conducted a single-institution study that retrospectively enrolled 72 patients with MBMs and found that the supplementation of ipilimumab with SRS not only improved tumor response, but also reduced edema volume. Furthermore, Acharya et al (61) demonstrated in another study that SRS treatment in combination with ICIs, regardless of whether ipilimumab or an anti-PD-1 agent was used, was associated with reduced local and distant intracranial failure compared with patients with MBMs treated with SRS alone.

In general, concurrent therapy, including RT and ICIs appears to be a promising treatment strategy for patients with BMs. However, the type of ICIs that results in the optimal treatment outcome when combined with RT has not been researched in detail. Choong et al (62) previously analyzed 108 patients with MBMs who were treated with SRS combined with various therapies, including anti-CTLA-4 $(n=28)$, anti-PD-1 $(n=11)$ and the B-Raf proto-oncogene inhibitor $(n=39)$. The median OS for patients treated with the anti-PD-1 agent + SRS (27.4 months) was found to be markedly higher compared with that for patients treated with anti-CTLA-4 agents + SRS (7.5 months). However, due to the number of patients treated with this type of immunotherapy being relatively small, the results of that study may not be representative of all patients with BMs.

The only prospective evidence of this modality of treatement comes from a phase I study conducted at Thomas Jefferson University (trial no. NCT01703507) (63). That study enrolled 16 patients to receive WBRT + ipilimumab $(n=5)$ or SRS + ipilimumab $(n=11)$ treatments, selected based on the intracranial disease burden. The results revealed that although concurrent treatment with ipilimumab + SRS or WBRT was well-tolerated, the efficacy of this combination treatment was not as expected, as 14/16 either exhibited disease progression and/or did not survive during the follow-up period. This could be due to the nature of phase I studies, in which treatment safety took precedence over efficacy as the primary endpoint of that study. Additionally, insufficient numbers of patients for the assessment of efficacy may have contributed to these unexpected results.

Following a review of the previous aforementioned studies, the concurrent use of combined RT and ICIs in treating BMs remains at an exploratory stage, as the efficacy of this combination has not yet been confirmed by a prospective phase II/III study. Furthermore, the optimal timing at which radiotherapy and immunotherapy should be administered is also a question that remains to be resolved. Although several retrospective studies $(58,59)$ have suggested that the concurrent application of RT and ICIs is associated with an improved OS compared with either treatment alone, the definition of 'concurrent' remains controversial. This is due to the fact that certain studies have reported this term to be the administration of ICIs as short as 2 weeks before or after RT (64), whilst others even extended this period to 4 weeks (65), even to as long as 5.5 months (66). However, a number of prospective trials are planned or are already underway to validate the use of ICIs combined with radiotherapy for the treatment of BMs, which should hopefully provide evidence to support the efficacy of this type of combinatorial therapy.

Safety of ICIs for the treatment of BMs. Immune-related adverse events (irAEs) are a series of inflammatory side effects caused by immunotherapy, most commonly affecting organs, including the gastrointestinal tract, endocrine glands and liver (67). In addition, the CNS can also be affected (68). Previous clinical trials have reported that irAEs of any grade can occur in 13-65\% patients who are treated with anti-PD-1 antibodies or ipilimumab monotherapy (36,69-73). Among these, neurological adverse events (AEs) caused by ICIs, including forms of hypophysitis, encephalitis, demyelinating polyneuropathy and encephalomyelitis, occur in $\sim 1 \%$ patients.

For patients with BMs, clinical data have demonstrated that the safety of ICIs was similar to that reported in patients with malignant tumors who did not have BMs (46). Furthermore, the most common AEs found were mainly systemic, including fatigue, diarrhea, headache and pruritus. In a clinical trial investigating the use of ipilimumab for the treatment of MBMs, the most commonly observed neurological AEs were grade $1 / 2$ headache and dizziness, with causes possibly related to ipilimumab treatment (35). In another clinical study, safety analysis of the results from a phase II study using pembrolizumab to treat patients with BMs revealed that neurological AEs occurred in $65 \%$ patients, with the most common neurological AEs being grade 1/2 gait disturbance (22\%) and headache (17\%) (39).

The nivolumab/ipilimumab combination appears to demonstrate a higher degree of toxicity compared with either nivolumab or ipilimumab alone. In a phase II ABC study, $63 \%$ patients receiving the nivolumab/ipilimumab combination presented with AEs that were grade 3 or higher. By contrast, in patients receiving nivolumab alone, only $16 \%$ patients reported such AEs (42). Safety data from the CheckMate-204 trial revealed that grade $3 / 4$ AEs occurred in $55 \%$ patients treated with combination therapy, including a patient who was afflicted with terminal immune-related myocarditis. Additionally, $8 \%$ patients experienced neurological AEs, half of which had to subsequently discontinue treatment as a result of the neurological AEs (46).

A common concern that is raised when combining ICIs and RT is the occurrence of possiby elevated or unexpected toxicity. Available clinical data suggest a weak association between the addition of brain radiotherapy to ICIs and the incidence or severity of irAEs. A recent large-scale retrospective study reported the outcomes of 260 patients with BMs who had 
received SRS and ICIs. That study demonstrated that the concurrent or sequential administration of SRS and ICIs did not increase acute toxicity. The rates of irAEs between patients who received concurrent SRS and ICI or either alone were not found to differ significantly (64). Apart from irAEs, the addition of ICI to RT was observed to markedly increase toxicity associated with RT, particularly neurotoxicity. From the previous studies aforementioned, the observed toxicities that can be potentially associated with RT in combined RT and ICI therapy included radiation necrosis ( $R N)$, neurocognitive decline and intratumoral hemorrhage (74-77). Among these, the most common side-effect was RN. Colaco et al (78) previously evaluated the safety of radiosurgery combined with various systemic therapies, including chemotherapy, target therapy and/or ICIs, in 180 patients with BMs from a number of primary malignancies. That study found that $\sim 37.5 \%$ patients who received ICIs developed RN, compared with $16.9 \%$ who received chemotherapy and $25.0 \%$ who received target therapy (78). In another study, Martin et al (79) evaluated the safety of 480 patients with BMs secondary to NSCLC, melanoma and RCC who were treated with SRS with/without ICIs. The results revealed that RN occurred in 20.0 and $6.85 \%$ patients who did and those who did not receive ICIs, respectively (79). These observations were consistent with those found in the studies conducted by Patel et al (80) and Kaidar-Person et al (81). These aforementioned studies raised significant concern that concurrent ICI and SRS treatment may increase the risk of RN. However, data from the subsequent studies did not exhibit the same trend, as neither Silk et al (82) nor Mathew et al (83) reported increased rates of $\mathrm{RN}$ in patients treated with the combination of RT and ipilimumab. Potential explanations for these disparities in results include the smaller cohort sizes among the studies and variations in the prescribed SRS doses, isodose line and number of fractions. Prospective studies that minimize these confounding factors are required to characterize the risks of combining RT and ICIs more comprehensively. The selected studies investigating the safety of ICI in BMs are presented in Table II.

Planned and ongoing clinical trials on ICIs on BMs. Although there is a paucity of prospective data validating the use of ICIs for the treatment of BMs, several prospective trials have been planned or are already underway (Table III). For example, NCT02460068 is a phase III trial that aims to investigate differences in the OS of patients with MBMs. Patients in that study will receive fotemustine, ipilimumab + fotemustine, or ipilimumab + nivolumab. In addition, NCT02681549 is a phase II study that will investigate the BM response rate in patients with BMs administered pembrolizumab and bevacizumab concurrently. NCT03903640 is a phase II study that will evaluate the efficacy of Optune, an FDA-approved device that delivers alternating electric fields to the tumor $(84,85)$, in comparison with ICIs in patients with BMs. Additional evidence of the efficacy and safety of ICIs for the treatment of BMs will be acquired from these studies.

\section{Cellular therapy for patients with BMs}

CAR T-cell therapy. CAR T cell therapy is a promising approach in immunotherapy that has been considered for the treatment of brain tumors. Using this technique, CAR T-cell receptors are specifically engineered to eradicate tumors through the recognition of surface proteins expressed on tumor cells (5). The extracellular domain of the CAR is the antibody region, allowing for recognition of the specific antigen in a MHC-independent manner (86). Inside the cell, the CAR contains a co-stimulatory signaling domain that is important for antitumor activity in addition to CAR T-cell proliferation and persistence (87). First-generation CARs contained an antigen-binding domain that is directly fused to the intracellular portion of $\mathrm{CD} 3 \xi$ chain of the T-cell receptor complex. However, this design demonstrated minimal clinical success due to the very low levels of engraftment observed in patients $(88,89)$. By contrast, second- and third-generation CARs included multiple co-stimulatory molecules from other receptors, including 4-1BB and CD28, in addition to the CD3 chain $(5,90)$, which markedly increased the antitumor efficacy and persistence of the CAR T-cells.

The efficacy of CAR T-cells for the treatment of hematological malignancies is impressive, particularly for acute lymphoblastic leukemia (ALL), where a clinical response rate of $90 \%$ was observed (91). This has resulted in numerous clinical studies on generating CARs directed against various hematological antigens, including CD19, CD20 and CD22 (92). For solid tumors, candidate target antigens currently being investigated in clinical trials in this manner include mesothelin, carcinoembryonic antigen, interleukin 13 receptor $\alpha$ (IL-13R $\alpha$ ) and human epidermal growth factor receptor 2 (93). This modular design, which provides the flexibility for adjusting antigen recognition and signaling domains based on the targeted cancer types, is one of the advantages of CARs (5).

Lee et al (94) previously conducted a phase I study to investigate the safety and efficacy of CD19-CAR T cells in the treatment of pediatric patients with ALL, which found that CD19-CAR T cells could be detected in the CSF (94). In 2016, Brown et al (95) reported a patient with multifocal glioblastoma achieving a notable response after being administered with intraventricular infusions of IL-13R $\alpha 2$ CAR T-cells (97). In addition, Abramson et al (96) described a patient with CNS diffuse large-B-cell lymphoma in 2017. After receiving CD19-CAR T-cell therapy, this patient achieved complete remission (96). These studies aforementioned suggest that systemically-administered CAR T-cells can reach the brain, which can be used in the treatment of brain tumors.

Initial clinical reports on the use of CAR T-cells for brain tumors have mainly focused on the treatment of recurrent or refractory glioblastoma. Several first-in-human studies CAR T-cell application in the treatment of glioblastoma have been published, including that of NCT00730613, which investigated the efficacy of intracranial IL13R $\alpha 2$-CAR delivery on glioblastoma (97), NCT02209376, which determined the safety and efficacy of EGFRvIII CAR-T for patients with glioblastoma (98) and NCT01109095, which assessed the safety and activity of human epidermal growth factor receptor 2 (HER2) CAR-T in adult and pediatric glioblastoma (99).

Studies focusing on using CAR T-cells for the treatment of solid tumors that have metastasized to the brain remain scant according to the literature. A single study was found, which was a preclinical study conducted by Priceman et al (100). 


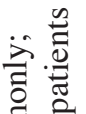

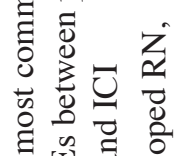

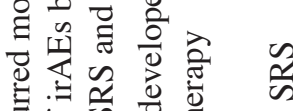

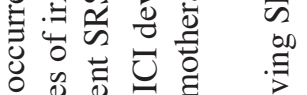

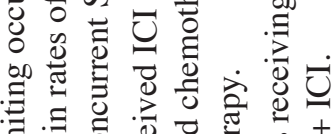
寻. $\begin{aligned} & 0 \\ & 0 \\ & 0\end{aligned}$

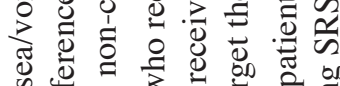

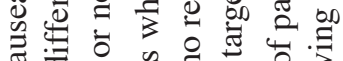

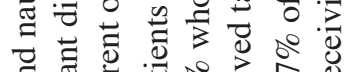

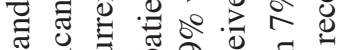
ণิ

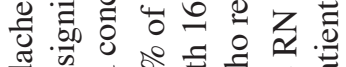

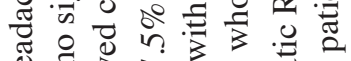
过 ๑ั .0

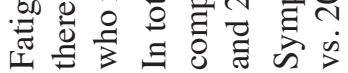

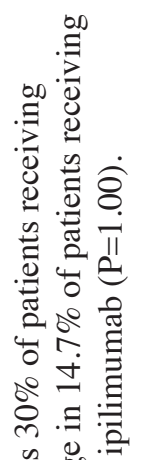

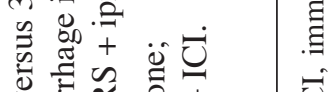

$>$ 造

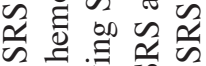

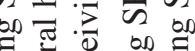
:

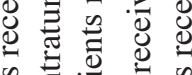
节节泀泀

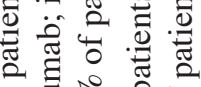

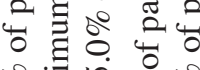
을

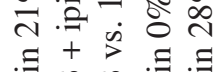

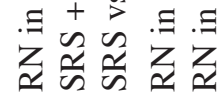

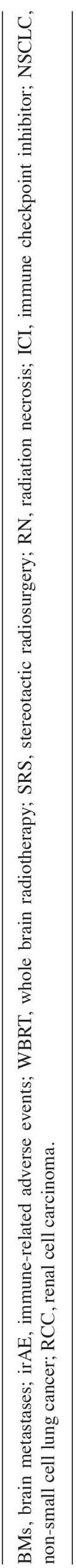


$\ddot{\tilde{m}}$ 蛋

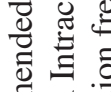
焉焉

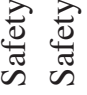

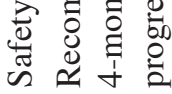

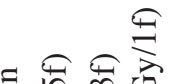

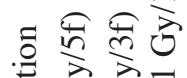

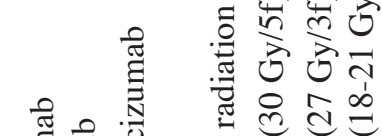

ڤ્ڤ

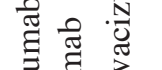

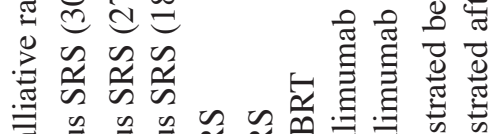

킁

:

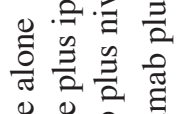

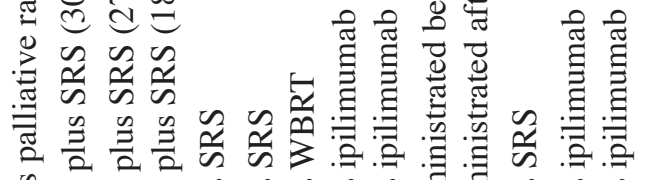

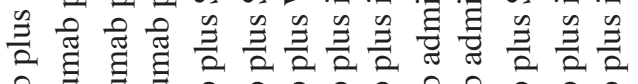

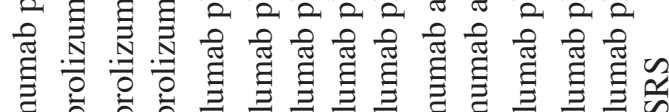

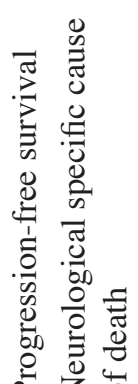

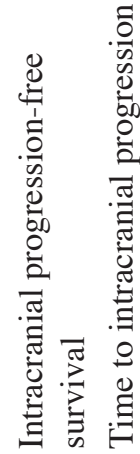

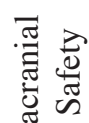
莺语

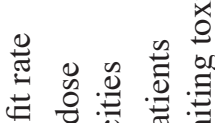

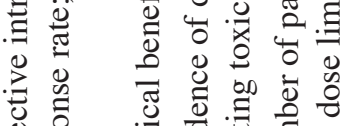

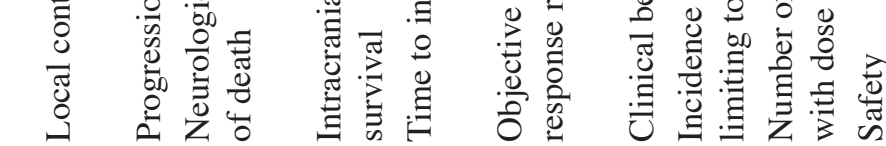

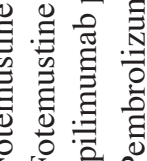

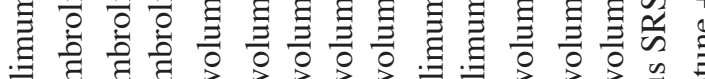

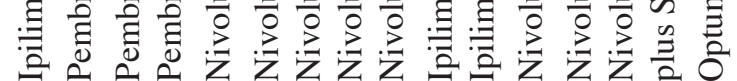<smiles>[C]1CCCCC1</smiles>

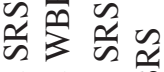
$=22$

$\infty$ in

규 \& $\infty$<smiles>[CH][CH]</smiles>

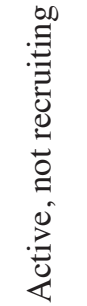

ㅇ $8 \stackrel{\infty}{\sim}$

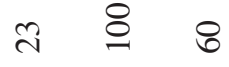

으 ते त

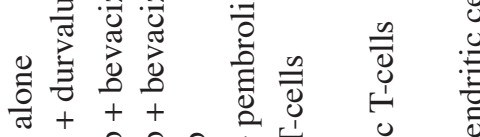

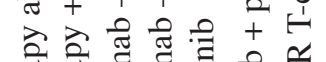

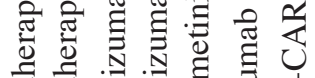

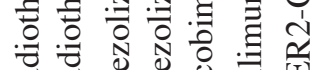

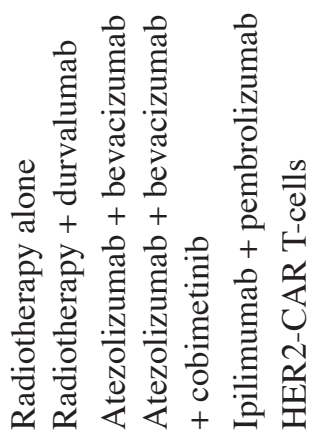

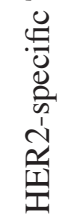

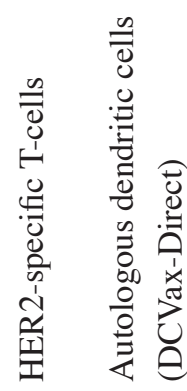<smiles>C1CCCCCCCCCCC1</smiles>

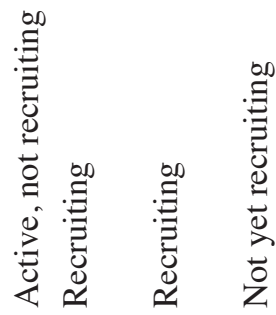

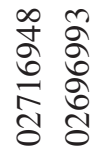

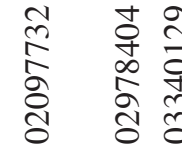

『

产

离离焉离

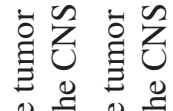

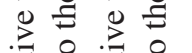

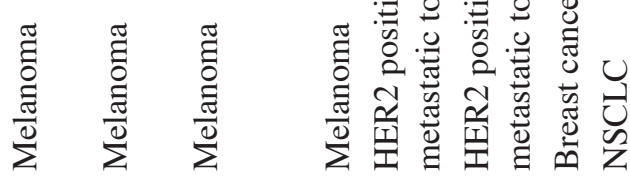

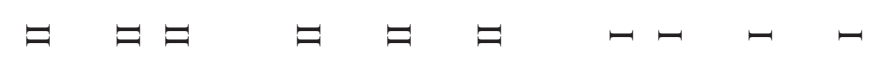

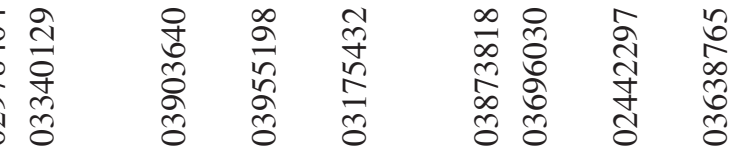


They used human breast cancer xenograft models that had metastasized to brain and evaluated the efficacy of HER2-CAR T-cells, which demonstrated robust antitumor responses mediated by HER2 CAR T-cells following local intratumor or regional intraventricular delivery (100).

At present, several clinical trials aiming to investigate the efficacy of CAR T-cells in treating solid metastatic brain tumors are underway. For example, NCT03696030 is a phase I study that will investigate the possible side effects and find the optimal dose of HER2-CAR T-cells for treating 39 patients with solid tumors that spread to the brain or leptomeninges. Additionally, NCT02442297 is a study that will be conducted by Baylor College of Medicine. It plans to enroll patients with recurrent or refractory HER2-positive primary CNS tumors or HER2-positive solid tumors that metastasized to the CNS, following which the safety and efficacy of HER2-CAR T cells will be evaluated (Table III). For patients with BMs, the potential therapeutic effect of CAR T-cells will be elucidated in the future.

Dendritic cell (DC) immunotherapy. DCs are professional APCs of the immune system that can capture antigens in the periphery and present them to T-cells (101). DCs can also facilitate the killing of tumor cells without affecting normal cells. Therefore, DC-based immunotherapy has been proposed as a promising cancer treatment method in various types of malignancies, including brain tumors (102-104).

Numerous clinical studies have investigated the safety and efficacy of DC-based immunotherapy in patients with glioblastoma patients. Liau et al (105) treated 12 patients with glioblastoma using DC vaccines (105), where the patients received 3 biweekly injections of $\leq 1 \times 10^{6} \mathrm{DCs}$ pulsed with 50-100 $\mu \mathrm{g}$ acid-eluted tumor peptides. These DC vaccinations were found to be well tolerated, where 6 patients developed measurable systemic antitumor CTL responses (105). Fadul et al (106) reported the outcomes of 10 patients with glioblastoma who were treated with tumor lysate-pulsed DCs (106). No severe adverse events were observed and the median OS was found to be 28 months for the vaccinated patients (106). Data involving patients with glioblastoma who were vaccinated with DCs utilizing specific tumor antigens have also been reported over the past number of years. Sampson et al (107) conducted a phase I dose escalation study to evaluate the safety and efficacy of DCs pulsed with an EGFRvIII peptide in patients with glioblastoma (107). Sakai et al (108) reported the results of 10 patients with recurrent malignant glioma who were treated with autologous DCs pulsed with peptides corresponding to the mutant Wilms' tumor 1 antigen. DCs pulsed with specific tumor antigens were demonstrated to be well tolerated, with promising results.

Although supportive data from clinical trials remain insufficient, a number of case studies have indicated the beneficial effects of DCs in patients with BMs. Laurell et al (109) conducted a phase I/II study to evaluate the ability of allogeneic DCs as immune enhancers for a patient with newly diagnosed metastatic RCC. In that study, a patient with brain and liver metastases was enrolled who responded well to the treatment, such that all brain and liver lesions completely disappeared (109). In another case study, 
Karbach et al (110) reported a patient with BMs associated with melanoma treated with radiosurgery and autologous tumor lysate-loaded DCs, the patient remained in the CR status 10 years following treatment (110).

Several ongoing studies will provide additional information on the effects of DCs on patients with BMs. NCT03638765 is a phase I study that will aim to evaluate the efficacy of DCVax-Direct, which is an autologously activated DC designed for intratumoral injection, in 24 patients with unresectable BMs from breast cancer and NSCLC. NCT02808416 is a phase I study that will to treat patients with BMs using personalized cellular tumor vaccines. In total, 10 patients will be immunized with DCs pulsed with mRNA-encoded tumor antigens. NCT01782274 and NCT01782287 are 2 clinical studies that will investigate the efficacy of proteome-based immunotherapy for treating breast and lung cancer BMs (Table III). In these 2 studies, a cohort of patients will receive a combination of the DC vaccine, allogeneic hematopoietic stem cells and cytotoxic lymphocytes, whilst the other cohort will be treated with either the DCs vaccine, autologous hematopoietic stem cells or cytotoxic lymphocytes alone.

\section{Conclusions}

Metastatic brain cancer involves several types of tumors and is typically associated with a poor prognosis. Since the concept of immune privilege in the CNS has been eroded, the advent of immunotherapy has opened a new avenue of potential therapeutic options for the treatment of patients with BMs. Although the BBB remains to be a significant obstacle, activated $T$ cells can circumvent this barrier. Tumor-specific $\mathrm{T}$ cells can be activated de novo during the development of cancer vaccines, oncolytic viral therapy and cell therapies engineered ex vivo. ICIs have demonstrated efficacy in BMs. Additionally, cellular therapies, including CAR T-cell therapy and DC vaccines, have been applied for the treatment of BMs. However, larger scale clinical trials, particularly those of phase III trials remain lacking. Therefore, the consequence of immunotherapy and/or radiotherapy on BMs largely remains unknown. The present article only reviewed immunotherapy against the BMs from melanoma, NSCLC and RCC, due to an insufficient number of studies on immunotherapy for BMs originating from other tumors, including gastrointestinal tumors and ovarian carcinoma. Ongoing, planned prospective trials and additional data are required to explore and further validate the effect of immunotherapy on BMs from a wider variety of malignant tumors.

\section{Acknowledgements}

Not applicable.

\section{Funding}

Not applicable.

\section{Availability of data and materials}

Not applicable.

\section{Authors' contributions}

RD, LC, ZS and TX made substantial contributions to the conception and design of the study and wrote the manuscript. QW, FJ and QP contributed to the study design and assisted in the literature search for this review article. All authors have read and approved the final version of the manuscript for publication.

\section{Ethics approval and consent to participate}

Not applicable.

\section{Patient consent for publication}

Not applicable.

\section{Competing interests}

The authors declare that they have no competing interests.

\section{References}

1. Tabouret E, Chinot O, Metellus P, Tallet A, Viens P and Gonçalves A: Recent trends in epidemiology of brain metastases: An overview. Anticancer Res 32: 4655-4662, 2012.

2. Kamath SD and Kumthekar PU: Immune Checkpoint Inhibitors for the Treatment of Central Nervous System (CNS) Metastatic Disease. Front Oncol 8: 414, 2018.

3. Hartgerink D, van der Heijden B, De Ruysscher D, Postma A, Ackermans L, Hoeben A, Anten M, Lambin P, Terhaag K, Jochems A, et al: Stereotactic Radiosurgery in the Management of Patients With Brain Metastases of Non-Small Cell Lung Cancer: Indications, Decision Tools and Future Directions. Front Oncol 8: 154, 2018.

4. Sevenich L: Turning 'Cold' Into 'Hot' Tumors-Opportunities and Challenges for Radio-Immunotherapy Against Primary and Metastatic Brain Cancers. Front Oncol 9: 163, 2019.

5. Akhavan D, Alizadeh D, Wang D, Weist MR, Shepphird JK and Brown CE: CAR T cells for brain tumors: Lessons learned and road ahead. Immunol Rev 290: 60-84, 2019.

6. Keskin DB, Anandappa AJ, Sun J, Tirosh I, Mathewson ND, Li S, Oliveira G, Giobbie-Hurder A, Felt K, Gjini E, et al: Neoantigen vaccine generates intratumoral $\mathrm{T}$ cell responses in phase Ib glioblastoma trial. Nature 565: 234-239, 2019.

7. Majzner RG, Theruvath JL, Nellan A, Heitzeneder S, Cui Y, Mount CW, Rietberg SP, Linde MH, Xu P, Rota C, et al: CAR T Cells Targeting B7-H3, a Pan-Cancer Antigen, Demonstrate Potent Preclinical Activity Against Pediatric Solid Tumors and Brain Tumors. Clin Cancer Res 25: 2560-2574, 2019.

8. Iorgulescu JB, Harary M, Zogg CK, Ligon KL, Reardon DA, Hodi FS, Aizer AA and Smith TR: Improved Risk-Adjusted Survival for Melanoma Brain Metastases in the Era of Checkpoint Blockade Immunotherapies: Results from a National Cohort. Cancer Immunol Res 6: 1039-1045, 2018.

9. Di Giacomo AM, Ascierto PA, Queirolo P, Pilla L, Ridolfi R, SantinamiM,Testori A,SimeoneE,GuidoboniM,Maurichi A, etal: Three-year follow-up of advanced melanoma patients who received ipilimumab plus fotemustine in the Italian Network for Tumor Biotherapy (NIBIT)-M1 phase II study. Ann Oncol 26: 798-803, 2015.

10. Lauko A, Thapa B, Venur VA and Ahluwalia MS: Management of Brain Metastases in the New Era of Checkpoint Inhibition. Curr Neurol Neurosci Rep 18: 70, 2018.

11. Sharpe AH and Pauken KE: The diverse functions of the PD1 inhibitory pathway. Nat Rev Immunol 18: 153-167, 2018.

12. Seidel JA, Otsuka A and Kabashima K: Anti-PD-1 and Anti-CTLA-4 Therapies in Cancer: Mechanisms of Action, Efficacy, and Limitations. Front Oncol 8: 86, 2018.

13. Goodman AM, Kato S, Bazhenova L, Patel SP, Frampton GM, Miller V, Stephens PJ, Daniels GA and Kurzrock R: Tumor Mutational Burden as an Independent Predictor of Response to Immunotherapy in Diverse Cancers. Mol Cancer Ther 16: 2598-2608, 2017. 
14. Omuro A,VlahovicG,LimM,SahebjamS,Baehring J,CloughesyT, Voloschin A, Ramkissoon SH, Ligon KL, Latek R, et al: Nivolumab with or without ipilimumab in patients with recurrent glioblastoma: Results from exploratory phase I cohorts of CheckMate 143. Neuro-oncol 20: 674-686, 2018.

15. Banks WA: From blood-brain barrier to blood-brain interface: New opportunities for CNS drug delivery. Nat Rev Drug Discov 15: 275-292, 2016.

16. Kipnis J: Multifaceted interactions between adaptive immunity and the central nervous system. Science 353: 766-771, 2016.

17. Sampson JH, Maus MV and June CH: Immunotherapy for Brain Tumors. J Clin Oncol 35: 2450-2456, 2017.

18. Engelhardt B, Vajkoczy P and Weller RO: The movers and shapers in immune privilege of the CNS. Nat Immunol 18: 123-131, 2017.

19. Preusser M, Lim M, Hafler DA, Reardon DA and Sampson JH: Prospects of immune checkpoint modulators in the treatment of glioblastoma. Nat Rev Neurol 11: 504-514, 2015.

20. El Rassy E, Farhat F and Kattan J: The forgotten role of adjuvant immune checkpoint inhibitors in preventing melanoma brain metastasis. Immunotherapy 10: 1289-1291, 2018

21. Koelzer VH, Rothschild SI, Zihler D, Wicki A, Willi B, Willi N, Voegeli M, Cathomas G, Zippelius A and Mertz KD: Systemic inflammation in a melanoma patient treated with immune checkpoint inhibitors-an autopsy study. J Immunother Cancer 4: 13, 2016.

22. Berghoff AS, Fuchs E, Ricken G, Mlecnik B, Bindea G, Spanberger T,Hackl M, Widhalm G, Dieckmann K, PrayerD, et al Density of tumor-infiltrating lymphocytes correlates with extent of brain edema and overall survival time in patients with brain metastases. OncoImmunology 5: e1057388, 2015.

23. Kluger HM, Zito CR, Barr ML, Baine MK, Chiang VL, Sznol M, Rimm DL, Chen L and Jilaveanu LB: Characterization of PD-L1 Expression and Associated T-cell Infiltrates in Metastatic Melanoma Samples from Variable Anatomic Sites. Clin Cancer Res 21: 3052-3060, 2015

24. Abate-Daga D, Ramello MC, Smalley I, Forsyth PA and Smalley KSM: The biology and therapeutic management of melanoma brain metastases. Biochem Pharmacol 153: 35-45, 2018.

25. Lehrer EJ, McGee HM, Peterson JL, Vallow L, Ruiz-Garcia H, Zaorsky NG, Sharma S and Trifiletti DM: Stereotactic Radiosurgery and Immune Checkpoint Inhibitors in the Management of Brain Metastases. Int J Mol Sci 19: 3054, 2018.

26. Darvin P, Toor SM, Sasidharan Nair V and Elkord E: Immune checkpoint inhibitors: Recent progress and potential biomarkers. Exp Mol Med 50: 1-11, 2018.

27. Eggermont AM, Chiarion-Sileni V, Grob JJ, Dummer R, Wolchok JD, Schmidt H, Hamid O, Robert C, Ascierto PA, Richards JM, et al: Adjuvant ipilimumab versus placebo after complete resection of high-risk stage III melanoma (EORTC 18071): A randomised, double-blind, phase 3 trial. Lancet Oncol 16: 522-530, 2015.

28. Leighl NB, Hellmann MD, Hui R, Carcereny E, Felip E, Ahn MJ, Eder JP, Balmanoukian AS, Aggarwal C, Horn L, et al: Pembrolizumab in patients with advanced non-small-cell lung cancer (KEYNOTE-001): 3-year results from an open-label, phase 1 study. Lancet Respir Med 7: 347-357, 2019.

29. Horn L, Spigel DR, Vokes EE, Holgado E, Ready N, Steins M, Poddubskaya E, Borghaei H,Felip E, Paz-Ares L, et al: Nivolumab Versus Docetaxel in Previously Treated Patients With Advanced Non-Small-Cell Lung Cancer: Two-Year Outcomes From Two Randomized, Open-Label, Phase III Trials (CheckMate 017 and CheckMate 057). J Clin Oncol 35: 3924-3933, 2017.

30. Larkin J, Hodi FS and Wolchok JD: Combined Nivolumab and Ipilimumab or Monotherapy in Untreated Melanoma. N Engl J Med 373: 1270-1271, 2015.

31. Brahmer J, Reckamp KL, Baas P, Crinò L, Eberhardt WE, PoddubskayaE,AntoniaS,PluzanskiA,Vokes EE,HolgadoE, etal: Nivolumab versus Docetaxel in Advanced Squamous-Cell Non-Small-Cell Lung Cancer. N Engl J Med 373: 123-135, 2015.

32. Topalian SLF, Hodi FS, Brahmer JR, Gettinger SN, Smith DC, McDermott DF, Powderly JD, Carvajal RD, Sosman JA, Atkins MB, et al: Safety, activity, and immune correlates of anti-PD-1 antibody in cancer. N Engl J Med 366: 2443-2454, 2012.

33. Lanier CM, Hughes R, Ahmed T, LeCompte M, Masters AH, Petty WJ, Ruiz J, Triozzi P, Su J, O'Neill S, et al: Immunotherapy is associated with improved survival and decreased neurologic death after SRS for brain metastases from lung and melanoma primaries. Neurooncol Pract 6: 402-409, 2019.
34. Rossi S, Finocchiaro G, Marchetti S, Toschi L and Santoro A: Checkpoint inhibitors: 'raising the bar' also in brain metastases from non-small-cell lung cancer? Immunotherapy 10: 403-410, 2018.

35. Margolin K, Ernstoff MS, Hamid O, Lawrence D, McDermott D, Puzanov I, Wolchok JD, Clark JI, Sznol M, Logan TF, et al: Ipilimumab in patients with melanoma and brain metastases: An open-label, phase 2 trial. Lancet Oncol 13: 459-465, 2012.

36. Di Giacomo AM, Ascierto PA, Pilla L, Santinami M, Ferrucci PF, GiannarelliD,Marasco A,RivoltiniL,SimeoneE,NicolettiSV,etal: Ipilimumab and fotemustine in patients with advanced melanoma (NIBIT-M1): An open-label, single-arm phase 2 trial. Lancet Oncol 13: 879-886, 2012

37. Queirolo P, Spagnolo F, Ascierto PA, Simeone E, Marchetti -P, Scoppola A, Del Vecchio M, Di Guardo L, Maio M, Di Giacomo AM, et al: Efficacy and safety of ipilimumab in patients with advanced melanoma and brain metastases. J Neurooncol 118: 109-116, 2014.

38. Goldberg SB, Gettinger SN, Mahajan A, Chiang AC, Herbst RS, Sznol M, Tsiouris AJ, Cohen J, Vortmeyer A, Jilaveanu L, et al: Pembrolizumab for patients with melanoma or non-small-cell lung cancer and untreated brain metastases: Early analysis of a non-randomised, open-label, phase 2 trial. Lancet Oncol 17: 976-983, 2016

39. Kluger HM, Chiang V, Mahajan A, Zito CR, Sznol M, Tran T, Weiss SA, Cohen JV, Yu J, Hegde U, et al: Long-Term Survival of Patients With Melanoma With Active Brain Metastases Treated With Pembrolizumab on a Phase II Trial. J Clin Oncol 37: 52-60, 2019.

40. Gandhi L, Rodríguez-Abreu D, Gadgeel S, Esteban E, Felip E, De Angelis F, Domine M, Clingan P, Hochmair MJ, Powell SF, et al; KEYNOTE-189 Investigators: Pembrolizumab plus Chemotherapy in Metastatic Non-Small-Cell Lung Cancer. N Engl J Med 378: 2078-2092, 2018.

41. Goldman JW, Crino L, Vokes EE, Holgado E, Reckamp K, Pluzanski A, SpigelD, Kohlhaeufl M, Garassino M, Chow LQ, etal: P2.36: Nivolumab (nivo) in Patients (pts) With Advanced (adv) NSCLC and Central Nervous System (CNS) Metastases (mets): Track: Immunotherapy. J Thorac Oncol Off Publ Int Assoc Study Lung Cancer 11: S238-S239, 2016.

42. Long GV, Atkinson V, Lo S, Sandhu S, Guminski AD, Brown MP, Wilmott JS, Edwards J, Gonzalez M, Scolyer RA, et al: Combination nivolumab and ipilimumab or nivolumab alone in melanoma brain metastases: A multicentre randomised phase 2 study. Lancet Oncol 19: 672-681, 2018.

43. Crinò L, Bronte G, Bidoli P, Cravero P, Minenza E, Cortesi E, Garassino MC, Proto C, Cappuzzo F, Grossi F, et al: Nivolumab and brain metastases in patients with advanced non-squamous non-small cell lung cancer. Lung Cancer 129: 35-40, 2019.

44. Bracarda S, Galli L, Maruzzo M, Lo Re G, Buti S, Favaretto A, Di Costanzo F, Sacco C, Merlano M, Mucciarini C, et al: Negative prognostic factors and resulting clinical outcome in patients with metastatic renal cell carcinoma included in the Italian nivolumab-expanded access program. Future Oncol 14: 1347-1354, 2018.

45. Gadgeel SM, Lukas RV, Goldschmidt J, Conkling P, Park K, Cortinovis D, de MarinisF, Rittmeyer A,Patel JD, vonPawel J, et al: Atezolizumab in patients with advanced non-small cell lung cancer and history of asymptomatic, treated brain metastases: Exploratory analyses of the phase III OAK study. Lung Cancer 128: 105-112, 2019.

46. Tawbi HA, Forsyth PA, Algazi A, Hamid O, Hodi FS, Moschos SJ, Khushalani NI, Lewis K, Lao CD, Postow MA, et al: Combined Nivolumab and Ipilimumab in Melanoma Metastatic to the Brain. N Engl J Med 379: 722-730, 2018.

47. Franceschini D, Franzese C, Navarria P, Ascolese AM, De Rose F, Del Vecchio M, Santoro A and Scorsetti M: Radiotherapy and immunotherapy: Can this combination change the prognosis of patients with melanoma brain metastases? Cancer Treat Rev 50: $1-8,2016$

48. Zindler JD, Slotman BJ and Lagerwaard FJ: Patterns of distant brain recurrences after radiosurgery alone for newly diagnosed brain metastases: Implications for salvage therapy. Radiother Oncol 112: 212-216, 2014

49. Golden EB, Pellicciotta I, Demaria S, Barcellos-Hoff MH and Formenti SC: The convergence of radiation and immunogenic cell death signaling pathways. Front Oncol 2: 88, 2012.

50. Ngwa W, Irabor OC, Schoenfeld JD, Hesser J, Demaria S and Formenti SC: Using immunotherapy to boost the abscopal effect. Nat Rev Cancer 18: 313-322, 2018. 
51. Lee Y, Auh SL, Wang Y, Burnette B, Wang Y, Meng Y, Beckett M, Sharma R, Chin R, Tu T, et al: Therapeutic effects of ablative radiation on local tumor require $\mathrm{CD} 8^{+} \mathrm{T}$ cells: Changing strategies for cancer treatment. Blood 114: 589-595, 2009.

52. Grass GD, Krishna N and Kim S: The immune mechanisms of abscopal effect in radiation therapy. Curr Probl Cancer 40: $10-24,2016$.

53. Reits EA,Hodge JW,Herberts CA, Groothuis TA, Chakraborty M, WansleyEK,Camphausen K,LuitenRM,deRuAH,NeijssenJ,etal: Radiation modulates the peptide repertoire, enhances MHC class I expression, and induces successful antitumor immunotherapy. J Exp Med 203: 1259-1271, 2006.

54. Matsumura S, Wang B, Kawashima N, Braunstein S, Badura M, CameronTO,BabbJS,SchneiderRJ,FormentiSC,DustinML, etal Radiation-induced CXCL16 release by breast cancer cells attracts effector T cells. J Immunol 181: 3099-3107, 2008.

55. Schaue D, Kachikwu EL and McBride WH: Cytokines in radiobiological responses: A review. Radiat Res 178: 505-523, 2012.

56. Sato H, Niimi A, Yasuhara T, Permata TBM, Hagiwara Y, Isono M, Nuryadi E, Sekine R, Oike T, Kakoti S, et al: DNA double-strand break repair pathway regulates PD-L1 expression in cancer cells. Nat Commun 8: 1751, 2017.

57. Herbst RS, Soria JC, Kowanetz M, Fine GD, Hamid O, Gordon MS, Sosman JA, McDermott DF, Powderly JD, Gettinger SN, et al: Predictive correlates of response to the anti-PD-L1 antibody MPDL3280A in cancer patients. Nature 515: 563-567, 2014.

58. Kiess AP, Wolchok JD, Barker CA, Postow MA, Tabar V, Huse JT, Chan TA, Yamada Y and Beal K: Stereotactic radiosurgery for melanoma brain metastases in patients receiving ipilimumab: Safety profile and efficacy of combined treatment. Int J Radiat Oncol Biol Phys 92: 368-375, 2015.

59. Cohen-Inbar O, Shih HH, Xu Z, Schlesinger D and Sheehan JP: The effect of timing of stereotactic radiosurgery treatment of melanoma brain metastases treated with ipilimumab. J Neurosurg 127: 1007-1014, 2017.

60. Diao K, Bian SX, Routman DM, Yu C, Kim PE, Wagle NA Wong MK, Zada G and Chang EL: Combination ipilimumab and radiosurgery for brain metastases: Tumor, edema, and adverse radiation effects. J Neurosurg 129: 1397-1406, 2018.

61. Acharya S, Mahmood M, Mullen D, Yang D, Tsien CI, Huang J, Perkins SM, Rich K, Chicoine M, Leuthardt E, et al: Distant intracranial failure in melanoma brain metastases treated with stereotactic radiosurgery in the era of immunotherapy and targeted agents. Adv Radiat Oncol 2: 572-580, 2017.

62. Choong ES, Lo S, Drummond M, Fogarty GB, Menzies AM, Guminski A, Shivalingam B, Clarke K, Long GV and Hong AM: Survival of patients with melanoma brain metastasis treated with stereotactic radiosurgery and active systemic drug therapies. Eur J Cancer 75: 169-178, 2017.

63. Williams NL, Wuthrick EJ, Kim H, Palmer JD, Garg S, Eldredge-Hindy H, Daskalakis C, Feeney KJ, Mastrangelo MJ, Kim LJ, et al: Phase 1 Study of Ipilimumab Combined With Whole Brain Radiation Therapy or Radiosurgery for Melanoma Patients With Brain Metastases. Int J Radiat Oncol Biol Phys 99: 22-30, 2017.

64. Chen L, Douglass J, Kleinberg L, Ye X, Marciscano AE, Forde PM, Brahmer J, Lipson E, Sharfman W, Hammers H, et al: Concurrent Immune Checkpoint Inhibitors and Stereotactic Radiosurgery for Brain Metastases in Non-Small Cell Lung Cancer, Melanoma, and Renal Cell Carcinoma. Int J Radiat Oncol Biol Phys 100: 916-925, 2018.

65. Qian JM, Yu JB, Kluger HM and Chiang VL: Timing and type of immune checkpoint therapy affect the early radiographic response of melanoma brain metastases to stereotactic radiosurgery. Cancer 122: 3051-3058, 2016.

66. An Y, Jiang W, Kim BYS, Qian JM, Tang C, Fang P, Logan J, D'Souza NM, Haydu LE, Wang XA, et al: Stereotactic radiosurgery of early melanoma brain metastases after initiation of anti-CTLA-4 treatment is associated with improved intracranial control. Radiother Oncol 125: 80-88, 2017.

67. Postow MA, Sidlow R and Hellmann MD: Immune-Related Adverse Events Associated with Immune Checkpoint Blockade. N Engl J Med 378: 158-168, 2018.

68. Weber JS, Hodi FS, Wolchok JD, Topalian SL, Schadendorf D, Larkin J, Sznol M, Long GV, Li H, Waxman IM, et al: Safety Profile of Nivolumab Monotherapy: A Pooled Analysis of Patients With Advanced Melanoma. J Clin Oncol 35: 785-792, 2017.

69. Nieder C and Hodi FS: Ipilimumab in patients with melanoma and brain metastases. Lancet Oncol 13: e277-e278, author reply e277-e278, 2012
70. Wolchok JD, Neyns B, Linette G, Negrier S, Lutzky J, Thomas L, Waterfield W, Schadendorf D, Smylie M, Guthrie T Jr, et al: Ipilimumab monotherapy in patients with pretreated advanced melanoma: A randomised, double-blind, multicentre, phase 2 , dose-ranging study. Lancet Oncol 11: 155-164, 2010.

71. Topalian SL, Sznol M, McDermott DF, Kluger HM, Carvajal RD, Sharfman WH, Brahmer JR, Lawrence DP, Atkins MB, Powderly JD, et al: Survival, durable tumor remission, and long-term safety in patients with advanced melanoma receiving nivolumab. J Clin Oncol 32: 1020-1030, 2014.

72. Robert C, Ribas A, Wolchok JDF, Hodi FS, Hamid O, Kefford R, Weber JS, Joshua AM, Hwu WJ, Gangadhar TC, et al: Anti-p rogrammed-death-receptor-1 treatment with pembrolizumab in ipilimumab-refractory advanced melanoma: A randomised dose-comparison cohort of a phase 1 trial. Lancet 384: 1109-1117, 2014.

73. Ribas A, Puzanov I, Dummer R, Schadendorf D, Hamid O, Robert C, Hodi FS, Schachter J, Pavlick AC, Lewis KD, et al: Pembrolizumab versus investigator-choice chemotherapy for ipilimumab-refractory melanoma (KEYNOTE-002): A randomised, controlled, phase 2 trial. Lancet Oncol 16: 908-918, 2015.

74. Anderson ES, Postow MA, Wolchok JD, Young RJ, Ballangrud Å, Chan TA, Yamada Y and Beal K: Melanoma brain metastases treated with stereotactic radiosurgery and concurrent pembrolizumab display marked regression; efficacy and safety of combined treatment. J Immunother Cancer 5: 76, 2017.

75. Liniker E, Menzies AM, Kong BY, Cooper A, Ramanujam S, Lo S, Kefford RF, Fogarty GB, Guminski A, Wang TW, et al: Activity and safety of radiotherapy with anti-PD-1 drug therapy in patients with metastatic melanoma. OncoImmunology 5: e1214788, 2016

76. Gerber NK, Young RJ, Barker CA, Wolchok JD, Chan TA, Yamada Y, Friguglietti L and Beal K: Ipilimumab and whole brain radiation therapy for melanoma brain metastases. J Neurooncol 121: 159-165, 2015.

77. Baskar R, Dai J, Wenlong N, Yeo R and Yeoh KW: Biological response of cancer cells to radiation treatment. Front Mol Biosci 1: 24, 2014

78. Colaco RJ, Martin P, Kluger HM, Yu JB and Chiang VL: Does immunotherapy increase the rate of radiation necrosis after radiosurgical treatment of brain metastases? J Neurosurg 125: $17-23,2016$

79. Martin AM, Cagney DN, Catalano PJ, Alexander BM, Redig AJ, Schoenfeld JD and Aizer AA: Immunotherapy and Symptomatic Radiation Necrosis in Patients With Brain Metastases Treated With Stereotactic Radiation. JAMA Oncol 4: 1123-1124, 2018.

80. Patel KR, Shoukat S, Oliver DE, Chowdhary M, Rizzo M, Lawson DH, Khosa F, Liu Y and Khan MK: Ipilimumab and Stereotactic Radiosurgery Versus Stereotactic Radiosurgery Alone for Newly Diagnosed Melanoma Brain Metastases. Am J Clin Oncol 40: 444-450, 2017.

81. Kaidar-Person O, Zagar TM, Deal A, Moschos SJ, Ewend MG, Sasaki-Adams D, Lee CB, Collichio FA, Fried D, Marks LB, et al: The incidence of radiation necrosis following stereotactic radiotherapy for melanoma brain metastases: The potential impact of immunotherapy. Anticancer Drugs 28: 669-675, 2017.

82. Silk AW, Bassetti MF, West BT, Tsien CI and Lao CD: Ipilimumab and radiation therapy for melanoma brain metastases. Cancer Med 2: 899-906, 2013

83. Mathew M, Tam M, Ott PA, Pavlick AC, Rush SC, Donahue BR, Golfinos JG, Parker EC, Huang PP and Narayana A: Ipilimumab in melanoma with limited brain metastases treated with stereotactic radiosurgery. Melanoma Res 23: 191-195, 2013.

84. Stupp R, Taillibert S, Kanner AA, Kesari S, Steinberg DM, Toms SA, Taylor LP, Lieberman F, Silvani A, Fink KL, et al: Maintenance Therapy With Tumor-Treating Fields Plus Temozolomide vs Temozolomide Alone for Glioblastoma: A Randomized Clinical Trial. JAMA 314: 2535-2543, 2015.

85. Wong ET, Lok E and Swanson KD: Clinical benefit in recurrent glioblastoma from adjuvant NovoTTF-100A and TCCC after temozolomide and bevacizumab failure: A preliminary observation. Cancer Med 4: 383-391, 2015.

86. Roselli E, Frieling JS, Thorner K, Ramello MC, Lynch CC and Abate-Daga D: CAR T Engineering: Optimizing Signal Transduction and Effector Mechanisms. BioDrugs: clinical immunotherapeutics, biopharmaceuticals and gene therapy. BioDrugs 33: 647-659, 2019.

87. Rice J, Nagle S, Randall J and Hinson HE: Chimeric Antigen Receptor T Cell-Related Neurotoxicity: Mechanisms, Clinical Presentation, and Approach to Treatment. Curr Treat Options Neurol 21: 40, 2019. 
88. Till BG, Jensen MC, Wang J, Chen EY, Wood BL, Greisman HA, Qian X, James SE, Raubitschek A, Forman SJ, et al: Adoptive immunotherapy for indolent non-Hodgkin lymphoma and mantle cell lymphoma using genetically modified autologous CD20-specific T cells. Blood 112: 2261-2271, 2008.

89. Kershaw MH, Westwood JA, Parker LL, Wang G, Eshhar Z, Mavroukakis SA, White DE, Wunderlich JR, Canevari S, Rogers-Freezer L, et al: A phase I study on adoptive immunotherapy using gene-modified $\mathrm{T}$ cells for ovarian cancer. Clin Cancer Res 12: 6106-6115, 2006.

90. Maus MV and June CH: Making Better Chimeric Antigen Receptors for Adoptive T-cell Therapy. Clin Cancer Res 22: 1875-1884, 2016.

91. Maude SL, Frey N, Shaw PA, Aplenc R, Barrett DM, Bunin NJ, Chew A, Gonzalez VE, Zheng Z, Lacey SF, et al: Chimeric antigen receptor $T$ cells for sustained remissions in leukemia. N Engl J Med 371: 1507-1517, 2014.

92. Holzinger A, Barden $\mathrm{M}$ and Abken $\mathrm{H}$ : The growing world of CAR T cell trials: A systematic review. Cancer Immuno Immunother 65: 1433-1450, 2016

93. Newick K, O'Brien S, Moon E and Albelda SM: CAR T Cell Therapy for Solid Tumors. Annu Rev Med 68: 139-152, 2017.

94.Lee DW, Kochenderfer JN, Stetler-Stevenson M, Cui YK, Delbrook C, Feldman SA, Fry TJ, Orentas R, Sabatino M, Shah NN, et al: T cells expressing CD19 chimeric antigen receptors for acute lymphoblastic leukaemia in children and young adults: A phase 1 dose-escalation trial. Lancet 385 : 517-528, 2015

95. Brown CE, Alizadeh D, Starr R, Weng L, Wagner JR, Naranjo A Ostberg JR, Blanchard MS, Kilpatrick J, Simpson J, et al: Regression of Glioblastoma after Chimeric Antigen Receptor T-Cell Therapy. N Engl J Med 375: 2561-2569, 2016.

96. Abramson JS, McGree B, Noyes S, Plummer S, Wong C, Chen YB, Palmer E, Albertson T, Ferry JA and Arrillaga-Romany IC: Anti-CD19 CAR T Cells in CNS Diffuse Large-B-Cell Lymphoma. N Engl J Med 377: 783-784, 2017.

97. Brown CE, Badie B, Barish ME, Weng L, Ostberg JR, Chang WC Naranjo A, Starr R, Wagner J, Wright C, et al: Bioactivity and Safety of IL13R $\alpha 2$-Redirected Chimeric Antigen Receptor $\mathrm{CD}^{+} \mathrm{T}$ Cells in Patients with Recurrent Glioblastoma. Clin Cancer Res 21: 4062-4072, 2015.

98. O'Rourke DM, Nasrallah MP, Desai A, Melenhorst JJ, Mansfield K, Morrissette JJD, Martinez-Lage M, Brem S, Maloney E, Shen A, et al: A single dose of peripherally infused EGFRvIII-directed CAR T cells mediates antigen loss and induces adaptive resistance in patients with recurrent glioblastoma. Sci Transl Med 9: eaaa0984, 2017.

99. Ahmed N, Brawley V, Hegde M, Bielamowicz K, Kalra M, Landi D, Robertson C, Gray TL, Diouf O, Wakefield A, et al: HER2-Specific Chimeric Antigen Receptor-Modified Virus-Specific T Cells for Progressive Glioblastoma: A Phase 1 Dose-Escalation Trial. JAMA Oncol 3: 1094-1101, 2017.
100. Priceman SJ, Tilakawardane D, Jeang B, Aguilar B, Murad JP, Park AK, Chang W-C, Ostberg JR, Neman J, Jandial R, et al: Regional Delivery of Chimeric Antigen Receptor-Engineered T Cells Effectively Targets HER2+ Breast Cancer Metastasis to the Brain. Clin Cancer Res 24: 95-105, 2018.

101. Reardon DA and Mitchell DA: The development of dendritic cell vaccine-based immunotherapies for glioblastoma. Semin Immunopathol 39: 225-239, 2017.

102. Li M, Han S and Shi X: In situ dendritic cell vaccination for the treatment of glioma and literature review. Tumour Biol 37 1797-1801, 2016.

103. Kyte JA, Mu L, Aamdal S, Kvalheim G, Dueland S, Hauser M, Gullestad HP, Ryder T, Lislerud K. Hammerstad H, et al: Phase I/II trial of melanoma therapy with dendritic cells transfected with autologous tumor-mRNA. Cancer Gene Ther 13: 905-918, 2006.

104. Antonios JP, Everson RG and Liau LM: Dendritic cell immunotherapy for brain tumors. J Neurooncol 123: 425-432, 2015.

105. Liau LM, Prins RM, Kiertscher SM, Odesa SK, Kremen TJ, Giovannone AJ,LinJW,Chute DJ,MischelPS,CloughesyTF, etal: Dendritic cell vaccination in glioblastoma patients induces systemic and intracranial T-cell responses modulated by the local central nervous system tumor microenvironment. Clin Cancer Res 11: 5515-5525, 2005

106. Fadul CE, Fisher JL, Hampton TH, Lallana EC, Li Z, Gui J, SzczepiorkowskiZM,TostesonTD, Rhodes CH,WishartHA, etal: Immune response in patients with newly diagnosed glioblastoma multiforme treated with intranodal autologous tumor lysate-dendritic cell vaccination after radiation chemotherapy. J Immunother 34: 382-389, 2011.

107. Sampson JH, Archer GE, Mitchell DA, Heimberger AB, Herndon JE II, Lally-Goss D, McGehee-Norman S, Paolino A, Reardon DA, Friedman AH, et al: An epidermal growth factor receptor variant III-targeted vaccine is safe and immunogenic in patients with glioblastoma multiforme. Mol Cancer Ther 8 : 2773-2779, 2009.

108. Sakai K, Shimodaira S, Maejima S, Udagawa N, Sano K, Higuchi Y, Koya T, Ochiai T, Koide M, Uehara S, et al: Dendritic cell-based immunotherapy targeting Wilms' tumor 1 in patients with recurrent malignant glioma. J Neurosurg 123: 989-997, 2015.

109. Laurell A, Lönnemark M, Brekkan E, Magnusson A, Tolf A, Wallgren AC, Andersson B, Adamson L, Kiessling R and Karlsson-Parra A: Intratumorally injected pro-inflammatory allogeneic dendritic cells as immune enhancers: A first-in-human study in unfavourable risk patients with metastatic renal cell carcinoma. J Immunother Cancer 5: 52-52, 2017.

110. Karbach J, Gnjatic S, Biskamp M, Atmaca A, Weidmann E, Brandt $\mathrm{K}$, Wahle C, Bernhard H, Knuth A and Jäger E: Long-term complete remission following radiosurgery and immunotherapy in a melanoma patient with brain metastasis: Immunologic correlates. Cancer Immunol Res 2: 404-409, 2014. 